\title{
The effect of flufenamic acid on gastrointestinal myoelectrical activity and transit time in dogs
}

\author{
G Farrugia, S Nitecki, G J Harty, M Camilleri, J H Szurszewski
}

\begin{abstract}
Background-Flufenamic acid, a fenamate, has been shown to alter markedly the membrane potential of small intestinal smooth muscle and increase intracellular calcium in single cells.

Aims-To determine the effects of flufenamic acid on myoelectrical motor activity and gastrointestinal transit in the intact animal.

Methods-Myoelectrical motor activity was recorded via seromuscular platinum electrodes sutured at regular intervals in the stomach and throughout the small intestine. Fasted and fed gastrointestinal transit was assessed using technetium$99 \mathrm{~m}\left({ }^{99 \mathrm{~m}} \mathrm{Tc}\right)$ as the radioactive marker linked to $1 \mathrm{~mm}$ amberlite pellets or added to the meal.

Results-Flufenamic acid (600 mg, intravenously) induced intense spike activity in the small intestine. The mean duration of irregular spike activity was 250 (7) minutes. Spike activity was more pronounced in the lower small intestine. Flufenamic acid also accelerated initial gastric emptying and markedly shortened transit time in the small intestine. In the fasted state the $50 \%$ transit time in the small intestine was 54 (8) minutes with infusion of flufenamic acid compared with 105 (10) minutes in the control group; in the fed state ${ }^{99 \mathrm{~m}} \mathrm{Tc}$ first reached the colon at 220 (10) minutes compared with 270 (12) minutes in the control group.

Conclusions-Flufenamic acid had marked effects on both myoelectrical motor complex activity and small intestinal transit in the dog. The observed effects suggest that flufenamic acid may be of potential use as a prokinetic agent.

(Gut 1998;42:258-265)
\end{abstract}

Keywords: prokinetic agents; transit studies; migrating motor complex

Flufenamic acid is a member of a group of compounds known as the fenamates. ${ }^{1}$ Other members of the group include niflumic acid, mefenamic acid, and meclofenamic acid. ${ }^{1}$ Fenamates have a wide variety of actions at both the clinical and cellular level. Clinically, fenamates are classified as non-steroidal antiinflammatory drugs (NSAIDs) and are used for the relief of pain and pyrexia. ${ }^{2}$ It is likely that the clinical mechanism of action of fenamates is through inhibition of the cyclooxygenase pathway. ${ }^{23}$ The therapeutic daily dose of fenamates varies from 200 to $1200 \mathrm{mg}^{2}$ and the most frequent side effects are referable to the gastrointestinal tract such as alteration in bowel habits, nausea, vomiting, and abdominal pain. ${ }^{14}$ About $30 \%$ of patients experience gastrointestinal side effects at therapeutic doses. ${ }^{4}$ The side effects are dose related and disappear on stopping the drug. ${ }^{4}$ A possible explanation for the gastrointestinal side effects of fenamates is that they alter gastrointestinal motility and transit. An increase in either contraction or relaxation would be expected to have significant effects on gastrointestinal intestinal motility and transit. An alteration in either the fasted pattern or fed pattern of myoelectrical activity of the gastrointestinal tract may be associated with an alteration in the time taken for substances to travel through the intestine. Non-steroidal anti-inflammatory drugs are known to alter gastrointestinal motility through inhibition of prostaglandin (PG) synthesis. Local synthesis of $\mathrm{PGE}_{2}$ and $\mathrm{PGF}_{2 \alpha}$ has been shown in human gastrointestinal smooth muscle. ${ }^{5}$ PGs have different effects on circular and longitudinal smooth muscle. In general PGE relaxes circular smooth muscle and contracts longitudinal smooth muscle while PGF first relaxes then contracts circular smooth muscle although several regional and species differences exist (reviewed in Bennet et $a l^{5}$ and Sanger and Bennet $\left.{ }^{6}\right)$. In humans the synthetic prostaglandin $\mathrm{E}_{1}$ analogue misoprostol decreases orocaecal transit time and has been proposed for treatment of slow transit constipation (reviewed in Farrugia et $a l^{7}$ ). Fenamates may therefore affect gastrointestinal smooth muscle through inhibition of PG synthesis. However, fenamates also have effects on membrane proteins. These effects appear to be unrelated to the inhibition of PG synthesis. At the cellular level, flufenamic acid interferes with the action of band three protein and therefore blocks anion transport in erythrocytes. $^{8}$

Fenamates are potent blockers of nonspecific cation channels especially when applied to the cytoplasmic site. ${ }^{910}$ Fenamates have also been found to inhibit calcium activated chloride channels in Xenopus oocytes $^{11}$ and to activate large conductance calcium activated potassium channels. ${ }^{12}$ In rabbit corneal epithelial cells, fenamates activate a whole cell potassium current ${ }^{13}$ and in human and canine jejunal circular smooth muscle cells, fenamates have two separate effects, one on outward potassium current and the membrane potential ${ }^{1415}$ and the other on intracellular calcium. ${ }^{16}$ Flufenamic acid markedly increases outward potassium currents and hyperpolarises the membrane potential. Based on this observation, one might expect that flufenamic acid would result in muscle
Accepted for publication 23 September 1997 
relaxation as the membrane potential would be driven negative to the threshold required for contraction. Indomethacin, another NSAID, did not mimic the cellular effects of fenamates on canine jejunal circular smooth muscle suggesting that the effects were mediated through a non-PG dependent pathway. At relatively low doses of oral flufenamic acid (200 mg) blood levels of the drug are between 20 and $30 \mu \mathrm{M} .{ }^{1}$ This is within the range for the effects on potassium currents, suggesting a possible causal relationship. The second cellular effect of flufenamic acid is on intracellular calcium. Flufenamic acid markedly increases intracellular calcium when applied at the dose of $500 \mu \mathrm{M} .^{16}$ An increase in intracellular calcium would be expected to cause smooth muscle contraction. It was therefore impossible to predict, based on single cell studies, what the effect of flufenamic acid would be in the intact animal.

The aim of the present study was to test the hypothesis that flufenamic acid stimulated myoelectrical activity and accelerated intestinal transit in both the fasting and fed state in the intact animal. The choice of flufenamic acid was based on the observation that it was the more efficacious fenamate in increasing jejunal circular smooth muscle whole cell current in vitro. ${ }^{14} 15$

\section{Methods}

Six female beagle dogs $(10-14 \mathrm{~kg})$ were used in this study. All six were used for studying the effect of flufenamic acid on the myoelectrical motor complex (MMC), and four dogs were also used for the transit studies. The research protocol was approved by the Institution's Animal Care and Use Committee. All dogs were previously adapted to the laboratory environment and were able to spend several hours in modified Pavlov slings without restraints. Dogs were housed in a specialised environment and were fed one $400 \mathrm{~g}$ can of Alpo beef chunks (Allentown, Pennsylvania) daily at a regular hour as well as ad lib dry lab chow. Water was available ad libitum. Under general anaesthesia, through a midline laparotomy incision all dogs had nine platinum monopolar electrodes sutured to the seromuscular layer of the gastrointestinal tract. One electrode was sutured to the stomach, two to the duodenum, and the remaining six at equal distance distributed throughout the remaining small intestine. The electrode wires were connected to a stainless steel cannula which was exteriorised through the left anterior abdominal wall. Following a recovery period of five to seven days, fasting and postprandial patterns of myoelectrical activity were recorded and characterised as previously described. ${ }^{17}$

RECORDING OF MYOELECTRICAL ACTIVITY

An eight channel recorder (Gould 2800S) was used to record myoelectrical activity. All data were also stored on an optical disk drive using custom written software programs to acquire, monitor, and store data. All dogs were fasted for 16 to 24 hours prior to each recording session. All six dogs were used for this portion of the study. Each dog was studied at least four times: fasted control, fasted drug $(600 \mathrm{mg})$, fed control, and fed drug $(600 \mathrm{mg})$. Additionally, in preliminary experiments, the dogs were also studied in the fasted state to determine a dose response curve for flufenamic acid $(75,150$, $200,300,600,1200 \mathrm{mg}$ ). Two experiments in different dogs were carried out for each dose. Higher doses were not tested due to difficulties in solubilising flufenamic acid which would have resulted in an unacceptable volume overload to the dogs of greater than 1 litre over two hours. To determine if the effects of flufenamic acid on myoelectrical motor complex were specific to the drug or were a more generalised feature of NSAIDs, the effects of indomethacin (250 mg), a commonly used NSAID, on myoelectrical activity were tested in two dogs. The usual human adult dose for indomethacin is up to $100 \mathrm{mg} /$ day. Each session lasted 8-12 hours in order to record three consecutive MMCs prior to either drug infusion or drug infusion and feeding. The control period of three consecutive MMCs was used to establish that the dog had a regular MMC pattern on the study day and to enable direct comparisons with the MMC activity recorded after drug and/or food administration. If the MMC pattern during the control period before drug infusion and/or feeding was irregular, the study was terminated and repeated on a different day. No dog was studied more than twice per week. To standardise drug delivery, flufenamic acid infusion was always started at the end of phase 3 of the MMC in the duodenal leads. The duration of each phase of the MMC was determined by direct visual measurement from the stored data using custom software. The start of irregular spike activity induced by flufenamic acid was defined as at least a fivefold increase from the amplitude of basal electrical activity. A motility index was used to quantify the effects of flufenamic acid on myoelectrical activity. The motility index was manually calculated as $\ln$ ([number of spikes $\times$ amplitude] +1$)$. A five minute period was used in calculating the motility index. For fasted studies the motility index was calculated immediately after the flufenamic acid infusion. For fed studies the motility index was calculated in the lower ileal lead for the five minute periods immediately prior to, and after flufenamic acid infusion. The postprandial pattern was established by a meal consisting of $400 \mathrm{~g}$ of beef chunks.

\section{TRANSIT STUDIES}

Four of the six dogs were used for gastrointestinal transit measurements. Transit studies were performed using technetium-99m $\left({ }^{99 \mathrm{~m}} \mathrm{Tc}\right)$ as the radioactive marker. ${ }^{18}{ }^{19}$ A GE starcam (General Electric, Syracuse, New York) gamma camera was used to measure isotope content in the gastrointestinal tract. Dogs were taught to sit with the abdomen apposed to the gamma camera and anterior isotope counts were recorded every 15 minutes. Using variable regions of interest, radioactivity was quantified in the stomach and small intestine. ${ }^{2021}$ Myoelectrical activity was also recorded during 
the transit studies to ensure that the ${ }^{99 \mathrm{~m}} \mathrm{Tc}$ was given at the same phase of the MMC for all dogs (end of phase 3 of the MMC in the duodenal leads). Fasting transit studies were performed using ion exchange pellets (Amberlite 410, Sigma, St Louis, Missouri) which were radiolabelled with $1 \mathrm{mCi}$ of ${ }^{99 \mathrm{~m}} \mathrm{Tc}$ pertechnetate. The isotope was placed in a gelatin capsule and the capsule was manually introduced into the posterior pharynx of the dog where it was swallowed and washed down with $5 \mathrm{ml}$ of water given via a syringe. This ensured that all the technetium reached the stomach at the same time. In the stomach the capsule was dissolved by the acidic environment. The onset of the study was defined as the time when the capsule was first seen in the stomach.

For the fed transit studies, similarly labelled ion exchange pellets were mixed with a scrambled egg which was then mixed with a regular meal ( $400 \mathrm{~g}$ of beef chunks) and the entire meal was consumed by the dogs. The start time was considered as the time when all the food was consumed (approximately 2.5 minutes). The study design was set up to study each dog four times: control fasted, drug fasted, control fed,

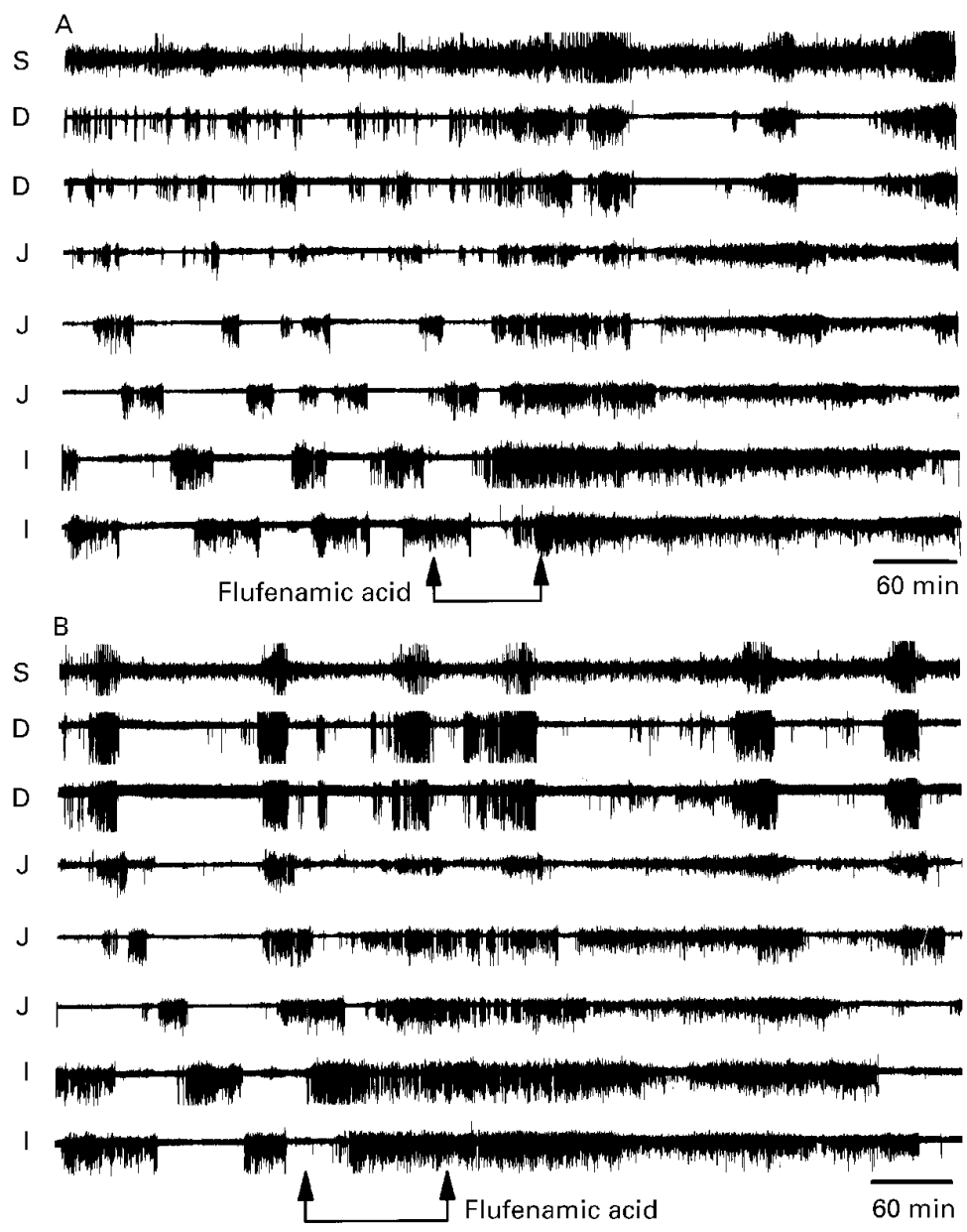

Figure 1 The effect of flufenamic acid ((A) $300 \mathrm{mg}$ in $250 \mathrm{ml}$ of normal Ringer's at 3 $\mathrm{ml} / \mathrm{min}$; (B) $600 \mathrm{mg} \mathrm{in} 500 \mathrm{ml}$ of normal Ringer's at $4.4 \mathrm{ml} / \mathrm{min}$ ) on the MMC recorded in separate experiments on the same dog. Data were recorded from one stomach (S) lead, two duodenal (D) leads, three jejunal (f) leads, and two ileal (I) leads. Twenty five minutes after the infusion was started, the normal MMC was replaced by intense spike activity. The effects of flufenamic acid were more noticeable in the distal leads where they appeared to last longer. The increase in spike activity lasted 5.5 hours in (A) and 7 hours in (B). and drug fed. However, one dog was only studied three times as the drug fed transit experiment could not be carried out due to loss of venous access. Flufenamic acid was infused over a 90 minute period. No dog was studied more than once within 48 hours and prior to feeding the radioactive markers a scan was always performed to ascertain that there were no ${ }^{99 \mathrm{~m}} \mathrm{Tc}$ counts left over from a previous experiment. Cobalt markers $\left({ }^{57} \mathrm{Co}, 5 \mu \mathrm{Ci}\right.$ encased in silastic tubing) were taped to each side of the dog at the level of the dog's xyphoid. These markers helped to identify the stomach, and filling of the small and large intestine as they provided a fixed point of reference to determine whether the dog moved during the experiment.

\section{TRANSIT DATA ANALYSIS}

Lag time and times for emptying of $25 \%, 50 \%$, and $90 \%$ of stomach contents were calculated. The lag time was defined as the time between the arrival of the capsule into the stomach and the time when $10 \%$ of the ${ }^{99 \mathrm{~m}} \mathrm{Tc}$ counts had emptied. The $50 \%$ transit time in the small intestine was calculated by subtraction of the time at which $50 \%$ of the ${ }^{99 \mathrm{~m}} \mathrm{Tc}$ had emptied from the stomach and the time when $50 \%$ of the ${ }^{99 \mathrm{~m}} \mathrm{Tc}$ counts had arrived in the colon. Time for first arrival of ${ }^{99 \mathrm{~m}} \mathrm{Tc}$ in the colon was also calculated.

\section{DRUGS}

Methohexital sodium ( $1 \mathrm{ml} / 5 \mathrm{lb}$ body weight) (Brevital, Lilly, Indianapolis, Indiana) was used intravenously for initiating anaesthesia. Halothane in oxygen (Halocarbon Lab, New Jersey) was supplied via a mechanical respirator to maintain anaesthesia during surgery. All dogs received an intramuscular injection of penicillin G, procaine plus benathaine (600 000 units, Flo-cillim, Bristol Laboratories, New York), prior to surgery. Flufenamic acid (Sigma, St Louis, Missouri) was dissolved in normal Ringer's solution and the $\mathrm{pH}$ adjusted to 7.35. Flufenamic acid was infused through a Harvard pump (Model 975) into the cephalic forelimb vein. Indomethacin was obtained from Merck, Sharp, and Dohme (West Point, Pennsylvania).

\section{STATISTICAL CALCULATIONS}

All values are expressed as mean (SEM). A paired $t$ test was used for statistical analysis. Values of p smaller than 0.05 were considered significant.

\section{Results}

GENERAL OBSERVATIONS

All dogs tolerated the infusion well (250$500 \mathrm{ml}$ ). No dogs had diarrhoea during or after the infusion of flufenamic acid and no vomiting, retching, or any other sign of discomfort was observed. The intravenous infusion resulted in urination either in the sling during recording of the myoelectrical motor complex or immediately after termination of the experiment. Infusion of normal Ringer's solution without flufenamic acid was without effect on myoelectrical activity. 




Figure 2 Lack of effect of indomethacin (250 mg, intravenously) on the MMC. Data were recorded from one stomach (S) lead, two duodenal (D) leads, three jejunal (f) leads, and two ileal (I) leads.
EFFECT OF FLUFENAMIC ACID ON THE

MYOELECTRICAL MOTOR COMPLEX

The most striking effect of intravenous infusion of flufenamic acid on the myoelectrical motor complex was the induction of spike activity which was more prominent in the lower jejunum and ileum than in the upper jejunum or duodenum (fig 1). Following infusion of flufenamic acid the motility index in the duodenum was $10.5(0.3)$ and in the ileum 11.1 (0.9) $(\mathrm{p}<0.05)$. Irregular spike activity was also noted in the stomach in $50 \%$ of experiments but was significantly less prominent than in the small intestine. Following infusion of flufenamic acid the motility index in the stomach was 10.1 (0.08) compared with $11.1(0.9)$ in the ileum $(p<0.05)$. Effects of flufenamic acid on myoelectrical activity were first noted at the $200 \mathrm{mg}$ intravenous infusion.

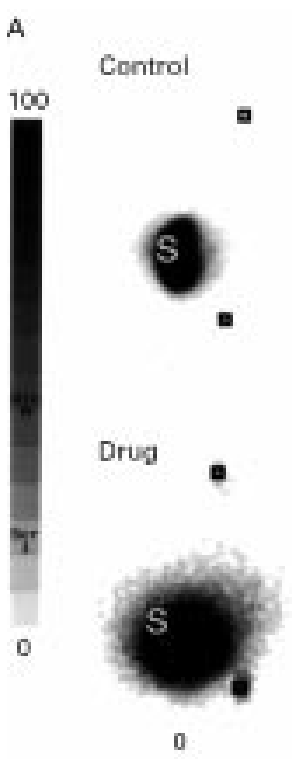

3

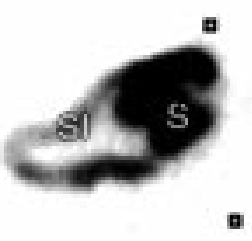

a

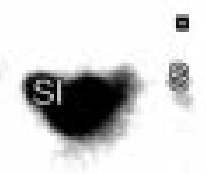



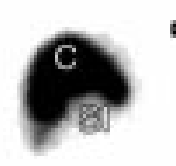

a

Headow

Time iminutes)



90

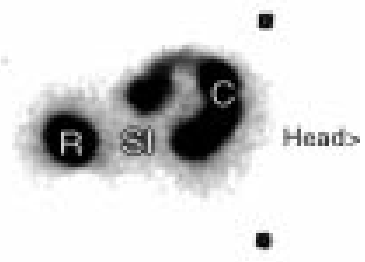

135

Time iminutes!

Control

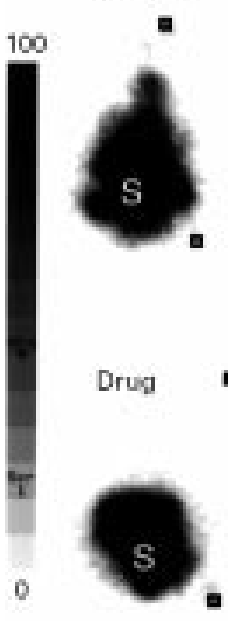

0

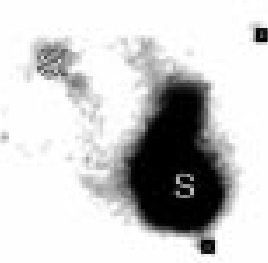

135
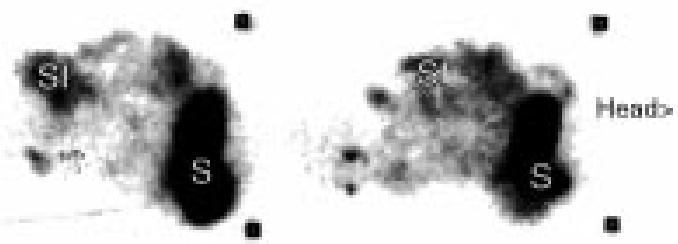

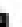

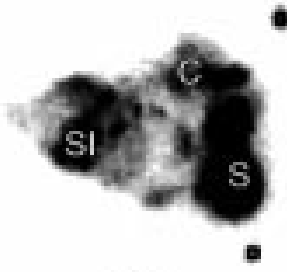

240

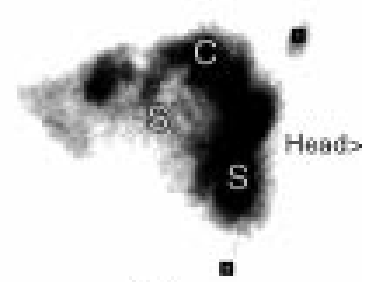

330

Figure 3 Representative examples of the effects of flufenamic acid (600 mg, intravenously) on transit time in the fasted $(A)$ and fed (B) dog (S, stomach; SI, small intestine; $C$, colon; $R$, rectum). 



3

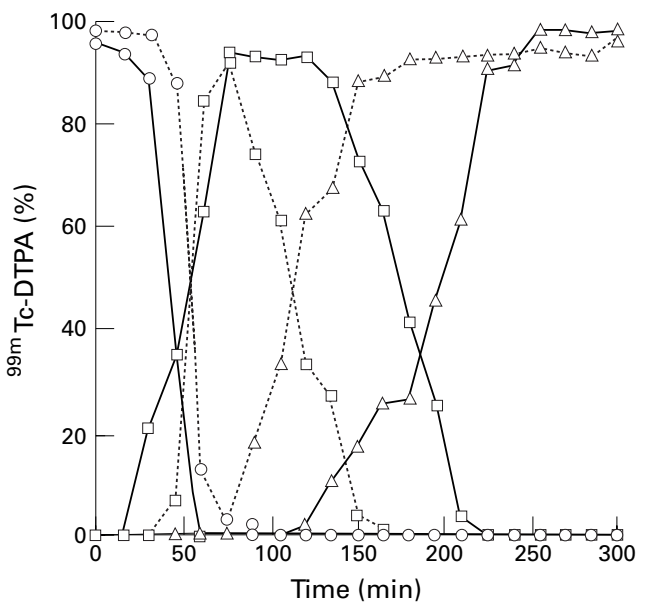

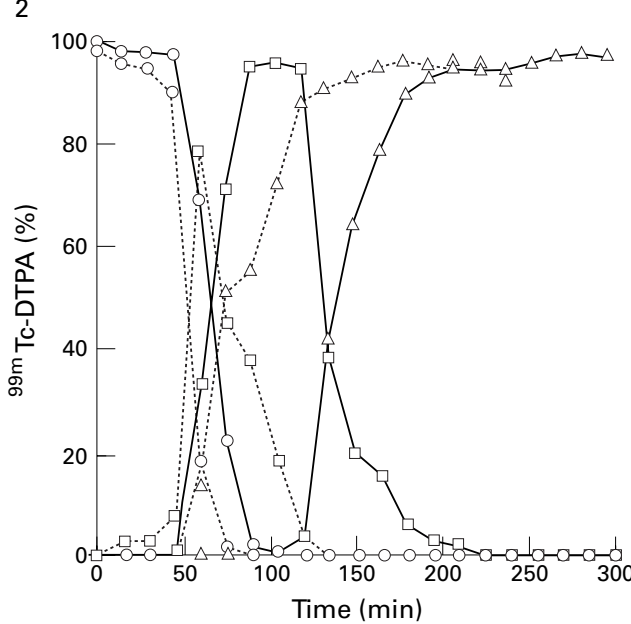

4

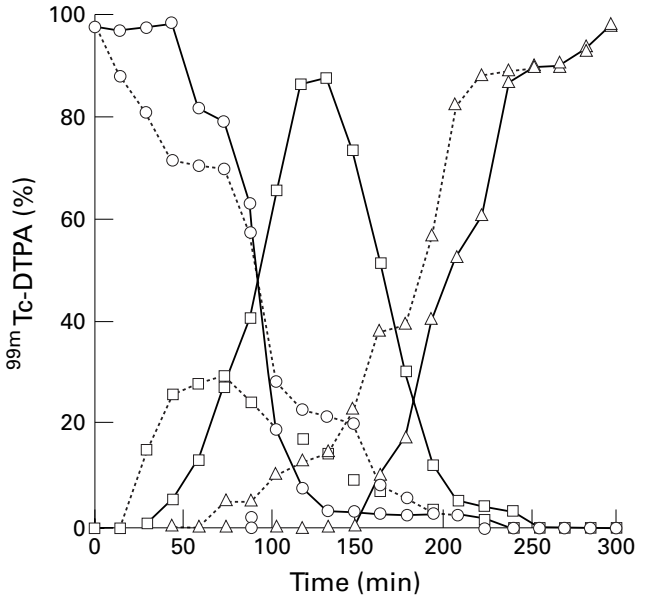

Figure 4 Plots of the ${ }^{99 m}$ Tc counts obtained for each of the four dogs in the fasted state (-, control; ...., drug).

No effects were noted at smaller doses $(75,150$ $\mathrm{mg}$ ). Increasing effects, quantified as the duration of irregular spike activity in the ileal leads, on small intestinal myoelectrical activity were noted with all tested doses above $200 \mathrm{mg}$ up to $1200 \mathrm{mg}$. Based on this preliminary dose response study, a dose of $600 \mathrm{mg}$ in $500 \mathrm{ml}$ was chosen for subsequent experiments as clear changes were seen in all dogs receiving this dose intravenously. The infusion rate was 2.2 $4.4 \mathrm{ml} /$ minute.

A lag time was present between the start of the infusion and the start of spike activity. The lag time for the effect of flufenamic acid depended on the infusion rate. At $2.2 \mathrm{ml} /$ minute, the mean lag time was 51.3 (2.3) minutes; at an infusion rate of $4.4 \mathrm{ml} /$ minute, it was 29.8 (1.2) minutes. The increase in regular spike activity could be interpreted as an increase in phase 2 of the myoelectrical motor complex as there was no evidence for a new myoelectrical motor complex interrupting this intense spike activity. As a result of the increase in spike activity, intravenous administration of flufenamic acid resulted in an increase in myoelectrical motor complex cycle time. Mean cycle time, defined as the time between the end of two consecutive periods of phase 3 activity in the duodenum, was 100.1 (3.1) minutes (24 MMCs analysed). After administration of normal Ringer's solution without the drug, cycle time was 101.4 (3) minutes (three dogs, 12 MMCs; normal Ringer's versus control period, $p>0.05$ ). In contrast, the mean cycle time during infusion of flufenamic acid (600 mg, intravenously) was 115.8 (3.7) (24 MMCs analysed; drug period versus control period, $\mathrm{p}<0.05)$.

The duration of spike activity induced by flufenamic acid as measured in the ileum from the end of the drug infusion was dose dependent. At $300 \mathrm{mg}$ flufenamic acid it was 173.1 (5.4) minutes; at $600 \mathrm{mg}, 249.6$ (7) minutes; and at $1200 \mathrm{mg}, 287.5$ (9.8) minutes.

A supratherapeutic dose of indomethacin (250 mg, intravenously, two dogs) had no effect on the spontaneously occurring myoelectrical motor complexes (fig 2). 
1



2

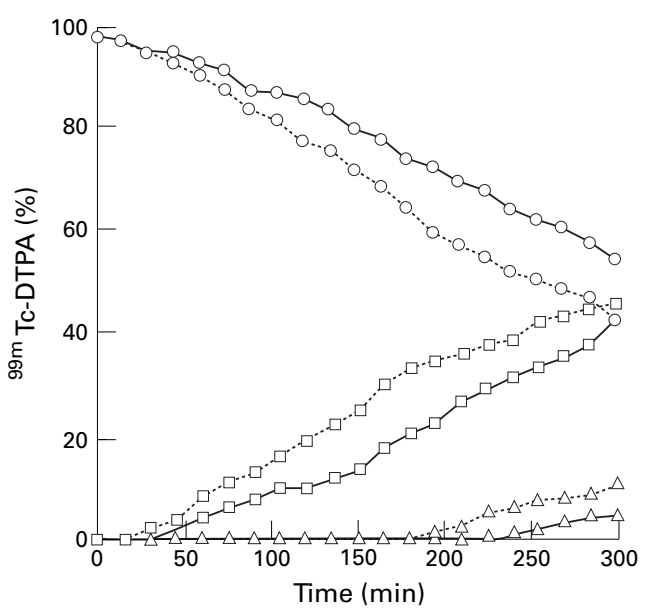

3

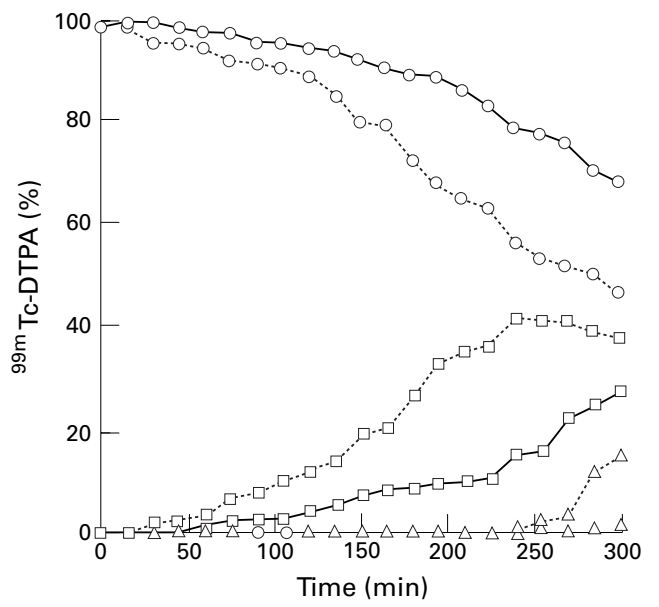

o Stomach

$\square$ Small intestine

$\triangle$ Colon

Figure 5 Plots of the ${ }^{99 m}$ Tc counts obtained for each of the three dogs in the fed state (-, control; ...., drug).

POSTPRANDIAL EFFECTS

The postprandial motility index, measured in the lower ileal lead, was $10.94(0.8)$ before, and $11.2(0.86)$ after the administration of flufenamic acid $(600 \mathrm{mg}$, intravenously, six dogs, $\mathrm{p}>0.05$, data not shown).

EFFECT OF FLUFENAMIC ACID ON TRANSIT TIMES Effect on fasting transit times

Stomach-In the stomach, the lag time was shortened from 57 (10) minutes in the control experiments to 32 (7) minutes during infusion of flufenamic acid ( $600 \mathrm{mg}$, intravenously, $\mathrm{n}=4$, $\mathrm{p}<0.05)$. The times for $50 \%$ and $90 \%$ of ${ }^{99 \mathrm{~m}} \mathrm{Tc}$ counts to leave the stomach during infusion of flufenamic acid were not statistically different from controls. The $50 \%$ emptying time was 89 (20) minutes for the control infusions and 76 (12) minutes for the flufenamic acid $(600 \mathrm{mg}$, intravenously) infusion ( $n=4, p>0.05)$ and the $90 \%$ emptying time was 107 (13) minutes for the control and 136 (22) minutes for the flufenamic acid infusion ( $\mathrm{n}=4, \mathrm{p}>0.05)$.

Small intestine-Flufenamic acid decreased small intestinal transit times in the fasted dogs. The $50 \%$ transit time in the small intestine was significantly shorter during administration of flufenamic acid (600 mg, intravenously) compared with controls (54 (8) minutes and 105
(10) minutes, respectively, $\mathrm{n}=4, \mathrm{p}<0.05$ ). Technetium was also detected earlier in the colon during infusion of flufenamic acid as compared with controls (75 (5) versus 161 (14) minutes, $n=4, p<0.05$ ). Figure $3 A$ shows the results of a typical fasting transit study. There was an increased number of ${ }^{99 \mathrm{~m}} \mathrm{Tc}$ counts in the small intestine 60 minutes after infusion of the drug. At 90 minutes, no ${ }^{99 \mathrm{~m}} \mathrm{Tc}$ was observed in the colon in the control experiment while ${ }^{99 \mathrm{~m}} \mathrm{Tc}$ had already arrived to the colon in the drug experiment, a reflection of a faster small intestinal emptying rate. At 135 minutes, most of the ${ }^{99 \mathrm{~m}} \mathrm{Tc}$ was still in the colon in the control experiment, while a significant portion of the ${ }^{99 m} \mathrm{Tc}$ had reached the rectum in the drug experiment. Figure 4 shows the plots of the ${ }^{99 \mathrm{~m}} \mathrm{Tc}$ counts for the four dogs.

\section{Effect on fed transit times}

Stomach-The lag time for gastric emptying was 152 (18) minutes in the control studies and 90 (7) minutes in dogs receiving intravenous flufenamic acid $(n=3, p<0.05)$. The time taken for $25 \%$ of the counts to leave the stomach was markedly altered by flufenamic acid (255 (20) minutes for the control group and 163 (7) minutes for the group receiving the drug; $n=3, p<0.05)$. A $50 \%$ 
emptying time was not calculated as feeding markedly prolonged gastric emptying in the control experiments.

Small intestine-Technetium was first observed in the small intestine after 50 (2.5) minutes in the control group $(n=3)$ and after $30(0)$ minutes in the group receiving flufenamic acid $(\mathrm{n}=3, \mathrm{p}<0.05,600 \mathrm{mg}$, intravenously), a reflection of the shorter stomach lag time. As feeding markedly prolonged transit times and $50 \%$ of the ${ }^{99 \mathrm{~m}} \mathrm{Tc}$ counts did not reach the colon during the study period, it was impossible to obtain a $50 \%$ emptying time for the small intestine. ${ }^{99 \mathrm{~m}} \mathrm{Tc}$ was first observed in the colon at 270 (12) minutes for the control group and 220 (10) minutes for the group receiving flufenamic acid $(n=3, p<0.05)$. Less than $10 \%$ of counts had reached the colon at 330 minutes (the last data point) in the control group. In contrast $10 \%$ of the counts were in the colon at 303 (7) minutes in the group receiving flufenamic acid $(n=3)$ suggesting that flufenamic acid also decreased small intestinal transit in the fed state. Figure $3 \mathrm{~B}$ shows the results of a typical fasting transit study. More ${ }^{99 \mathrm{~m}} \mathrm{Tc}$ counts had reached the small intestine at 240 minutes in the drug experiments compared with the control experiment. Also, at 240 minutes, ${ }^{99 \mathrm{~m}} \mathrm{Tc}$ had reached the colon in the drug experiment while none had reached the colon in the control experiment. At 330 minutes, more ${ }^{99 \mathrm{~m}} \mathrm{Tc}$ had reached the colon in the drug experiment while again none had reached the colon in the control experiment. Figure 5 shows the plots of the ${ }^{99 \mathrm{~m}} \mathrm{Tc}$ counts for the three dogs.

\section{Discussion}

The present study showed that flufenamic acid was a potent stimulator of smooth muscle contraction in vivo. Application of flufenamic acid resulted in an increase in irregular spike activity that lasted for a considerable period after the drug infusion was stopped. The effects of flufenamic acid appeared to be somewhat regional as greater effects were seen in the lower portion of the small intestine as compared with the duodenum and upper jejunum. An increase in irregular spike activity would not necessarily result in a decreased transit time as increased spike activity may lead to segmental contractions rather than coordinated movement. The second portion of our study was therefore designed to test the effect of flufenamic acid on gastrointestinal transit using a previously validated technique. ${ }^{18}{ }^{19}$ The results of the transit studies showed that flufenamic acid was a potent prokinetic drug, accelerating initial gastric emptying and markedly shortening small intestinal transit time. The effects of flufenamic acid were observed both in the fasted state and the fed state, suggesting that flufenamic acid may potentially be of benefit in disorders of motility, such as intestinal pseudo-obstruction, which is characterised by increased gastrointestinal transit times. Clearly, this would need to be rigorously tested in a clinical trial. This is not feasible in the United States as flufenamic acid is currently not licensed for human use; mefenamic acid and meclofenamic acid are the only fenamates licensed for clinical use in the USA. We have tested the effects of mefenamic acid (600 mg, intravenously) on myoelectrical activity in the dog (data not shown). Although mefenamic acid also altered the myoelectrical motor complex inducing a spiking activity similar to that seen with flufenamic acid, the effects were weaker and of shorter duration.

Another potential problem with the use of fenamates as prokinetic agents is that they are NSAIDs. They therefore have the same side effect profile as other NSAIDs and have the potential for causing gastric erosions and ulcers. ${ }^{4}$ If a subsequent human clinical trial were to show potential benefits for the use of flufenamic acid or other fenamates as prokinetic agents it may be advantageous to alter their structure to reduce their side effect profile. Potential modifications include replacing the benzoyl group that could be responsible for ulceration with a sulphonic acid containing phenyl group. Hybrids of fenamates can also be produced with compounds that do not significantly cause ulceration, such as NS-398. ${ }^{22}$ The observed effects of flufenamic acid appear to be limited to the fenamate group and not applicable to other NSAIDs. Indomethacin, another NSAID, had no effect on migratory motor complex activity even when used at doses much higher than usual human adult therapeutic doses. Therefore, the effects of flufenamic acid on gastrointestinal transit cannot be generalisable to other NSAIDs. The data also suggest that the mechanism of action of flufenamic acid on intestinal transit was not through the PG pathway as indomethacin, a cyclooxygenase inhibitor, was ineffective in altering myoelectrical activity.

In summary, flufenamic acid had marked stimulatory effects on both myoelectrical motor complex activity as well as intestinal transit, accelerating early gastric emptying and markedly reducing small intestine transit times in both the fasted and fed states. Flufenamic acid may therefore be of potential use as a prokinetic agent. This hypothesis will require future clinical studies in humans.

The authors thank Louis J Kost and Gary Stoltz for technical assistance, Erika Wohlfiel and Jan Applequist for secretarial assistance, and Dr S F Phillips for helpful comments and for the use of the gamma camera facilities. This work was supported by DK17238 and by an AGA Industry Research Scholar Award (GF).

1 Glazko AJ. Experimental observations of flufenamic, mefenamic and meclofenamic acids. Part III. Metabolic disposition, in fenamates in medicine. Ann Phys Med Suppl disposition, in fena.

2 Hart FD. Treatment of the arthropathies with antiinflammatory compounds. In: Williamson NWR, ed. Antiinflammatory compounds. New York: Dekker, 1987:23-86.

3 Terada H, Muraoka S, Fujita T. Structure-activity relationships of fenamic acids. $\mathcal{F}$ Med Chem 1974;17:330-4.

4 Oradell NJ. Physicians desk reference. Medical Economics, 1989:1590.

5 Bennet A, Hensby G, Sanger GJ, Stamford IF. Metabolites of arachidonic acid formed by human intestinal tissues and $435-44$

6 Sanger GJ, Bennet A. Regional differences in the responses to prostanoids of circular muscle from guinea-pig isolated intestine. F Pharm Pharmacol 1980;32:705-8.

7 Farrugia G, Camilleri M, Whitehead W. Therapeutic strategies for motility disorders. Gastroenterol Clin North Am 1996;25:225-46.

8 Knauf PA, Mann NA. Use of niflumic acid to determine the nature of the asymmetry of the human erythrocyte anion exchange system. $\mathcal{F}$ Gen Physiol 1984;83:703-25. 
9 Gogelein H, Dahlem D, Englert HC, Lang HJ. Flufenamic acid, mefenamic acid and niflumic acid inhibit single nonselective cation channels in the rat exocrine pancreas. FEBS Lett 1990;268:79-82

10 Gogelein H, Pfannmuller B. The nonselective cation channel in the basolateral membrane of rat exocrine pancreas. Pflugers Arch 1989;413:287-98.

11 White MM, Aylwin M. Niflumic and flufenamic acids are potent reversible blockers of $\mathrm{Ca}^{2+}$-activated $\mathrm{Cl}^{-}$channels in Xenopus oocytes. Mol Pharmacol 1990;37:720-4.

12 Ottolia $M$, Toro L. Potentiation of large conductance $\mathrm{K}_{\mathrm{C}}$ channels by niflumic, flufenamic, and mefenamic acids. Biophys 7 1994;67:2272-9.

13 Rae JL, Farrugia G. A whole cell potassium current in rabbit corneal epithelium activated by fenamates. $7 \mathrm{Membr}$ Biol 1992;129:81-97.

14 Farrugia G, Rae JL, Szurszewski JH. Characterization of a delayed rectifier in canine jejunal circular smooth muscle and its activation by fenamates. F Physiol 1993;468:297-310.

15 Farrugia G, Rae JL, Sarr M, Szurszewski JH. A potassium whole cell current in jejunal human circular smooth whole cell current in jejunal human

16 Nitecki S, Farrugia G, Rich A, Szurszewski JH. Effects of flufenamic acid in vivo on MMC activity and in vitro on intracellular calcium in the canine lower small intestine. Gastroenterology 1994;107:1226.

17 Nitecki S, Karmeli R, Harty GJ, Kamei C, Yaksh TL, Szurszewski JH. Long-term perfusion of the cerebroventricular system of dogs without leakage to the peripheral circulation. Am f Physiol 1994;267:R1309-19.

18 Camilleri M, Zinsmeister AR, Greydanus MP, Brown ML, Proano $M$. Towards a less costly but accurate test of gastric emptying and small bowel transit. Dig Dis Sci 1991;36:60915.

19 von der Ohe MR, Camilleri M. Measurement of small bowel and colonic transit: indications and methods. Mayo Clin Proc 1992;67:1169-79.

20 Wen J, Phillips SF, Kost L. Scintigraphic measurement of regional gut transit in the dog [abstract]. Gastroenterology 1995;108(suppl):A709.

21 Nguyen A, Camilleri M, Kost LJ, et al. SDZ HTF919 stimulates canine colonic motility and transit in vivo. $\mathcal{F}$ Pharmacol Exp Ther 1997;280:1270-6.

22 Arai I, Hamasaka Y, Futaki N, et al. Effect of NS-398, a new nonsteroidal anti-inflammatory agent, on gastric ulceration and acid secretion in rats. Res Commun Chem Pathol and acid secretion in rats.
Pharmacol 1993;81:259-70. 